Trinity University

Digital Commons @ Trinity

Psychology Faculty Research

Psychology Department

6-2019

\title{
Training to Inhibit Negative Content Affects Memory and Rumination
}

Shimrit Daches

Nilly Mor

Paula T. Hertel

Trinity University, phertel@trinity.edu

Follow this and additional works at: https://digitalcommons.trinity.edu/psych_faculty

Part of the Psychology Commons

Publication Details

Cognitive Therapy and Research

\section{Repository Citation}

Daches, S., Mor, N., \& Hertel, P. (2019) Training to inhibit negative content affects memory and rumination. Cognitive Therapy and Research, 43(3), 1018-1027. doi: 10.1007/s10608-019-10023-0

This Pre-Print is brought to you for free and open access by the Psychology Department at Digital Commons @ Trinity. It has been accepted for inclusion in Psychology Faculty Research by an authorized administrator of Digital Commons@ Trinity. For more information, please contact jcostanz@trinity.edu. 


\title{
Training to Inhibit Negative Content affects Memory and Rumination
}

\begin{abstract}
Depressive rumination, the tendency to engage in repetitive self-focus in response to distress, seems to be affected by a variety of cognitive biases that in turn maintain negative emotional states. The current study examined whether the difficulty in inhibiting attention to negative information contributes to rumination and to rumination-related biases in memory. Seventy-nine ruminators underwent a 3-week computer-based training, designed to increase either inhibition of negative words or attention to them. On immediate post-training trials, as well as on 2-week follow-up tests, we found evidence for transfer of inhibition training. Training effects also occurred on session-by-session and post-training measures of state rumination, but not on a measure of trait rumination, assessed two weeks later. Finally, participants who were trained to inhibit negative material subsequently showed less negative bias on a memory test. These findings further establish the causal role of biased inhibition in rumination, and substantiate the view of rumination as a habit that encourages people to perceive, interpret, and remember events in a repetitive self-focused manner. 167 words
\end{abstract}

Keywords: rumination, inhibition, memory, cognitive-bias modification, depression 


\section{Training to Inhibit Negative Content affects Memory and Rumination}

Some people habitually respond to negative mood by dwelling on it rather than focusing on solutions that would make things better. Moreover, people who ruminate are more prone to developing psychopathology (particularly depression); they recover more slowly; and they benefit less from therapy (see reviews by Aldao, NolenHoeksema, \& Schweizer, 2010; Nolen-Hoeksema, Wisco, \& Lyubomirsky, 2008). In response, a body of research has developed to examine the underlying mechanisms of rumination that might suggest methods for its reduction.

Cognitive models of rumination point to deficient cognitive control (e.g., Hertel, 1997; Koster, De Lissnyder, Derakshan, \& De Raedt, 2011; Linville, 1996; Whitmer \& Gotlib, 2013). Specifically, research has revealed rumination-related biases in inhibitory processes. Such biases are thought to impair the ability to resist distraction from irrelevant negative stimuli in the environment and interference from negative thoughts (e.g., Friedman \& Miyake, 2004; Hasher \& Zacks, 1988). These biases may disrupt processing of important goal-relevant information. By doing so, these inhibitory biases further enhance the habit of dwelling on the negative, and impede the development of new and adaptive habits (Hertel, 2004). Impaired inhibition of negative content - revealed by comparing the performance of ruminators and othershas been well documented (See Yang, Cao, Shields, Teng, \& Liu, 2017, for a metaanalysis). Prospective research has also shown that a poor ability to inhibit (Zetsche \& Joormann, 2011) or shift to and from negative content (Demeyer, De Lissnyder, Koster, \& De Raedt, 2012), predicts rumination 6-12 month later.

Given the suggested importance of impaired cognitive control in rumination, several studies have used cognitive-bias modification (CBM) procedures to ameliorate these impairments (see reviews by Koster, Hoorelbeke, Onraedt, Owens, \& 
Derakshan, 2017; Mor \& Daches, 2015). In CBM, a putative cognitive bias is targeted with the aim of affecting emotional symptoms. CBM procedures have been fruitful in elucidating the role of cognitive biases in emotional disorders (e.g., Beevers, Clasen, Enock, \& Schnyer, 2015), and in delineating causal pathways between these biases and rumination (e.g., Hirsch et al., 2018; Vermeulen, Brown, Raes, \& Krans, 2018). Similarly, CBM procedures can shed light on the role that impaired inhibition plays in rumination. Moreover, to the extent that inhibition deficits and biases contribute to emotional disorders, focused training may be a promising venue for prevention.

To examine the possibility that training might contribute to prevention, we (Daches \& Mor, 2014) conducted a training experiment based on the negative affective priming (NAP) paradigm. We assigned ruminators to four sessions of training to either inhibit or attend to negative stimuli, or to a sham training condition in which the same negative stimuli were presented but no inhibition was required. The NAP was originally designed to evaluate the strength of inhibitory processes by measuring latencies to categorize the emotional valence of targets that had each served as the non-attended distractor on the previous trial (e.g., Joormann, 2006). On each training trial in our experiment, participants saw two words (a target and a distractor, each identified by a different color) and indicated the valence of the target while ignoring the distractor. In the attend-negative condition, the target was negative on most trials, whereas in the inhibit-negative condition, the distractor was negative on most trials. On a subsequent transfer task (with equal numbers of each trial type), the data from the attend-negative training condition revealed reduced inhibition of negative stimuli whereas those from the ignore-negative condition revealed increased inhibition. Compared to participants in the attend-negative condition, those in the 
inhibit-negative condition took longer to respond to negative targets on the transfer test, and they also produced lower scores on a trait rumination inventory.

In addition to their negative attentional bias, ruminators also experience negative memory bias (Engen \& Anderson, 2018). Several studies have linked memory biases to ruminative thinking. For example, ruminators retrieve negative autobiographical memories (Lyubomirsky, Caldwell, \& Nolen-Hoeksema, 1998) and negative selfrelated material (Moulds, Kandris, \& Williams, 2007) more readily than do nonruminators, and they have difficulty forgetting negative events (Dieler, Herrmann, \& Fallgatter, 2014; Hertel \& Gerstle, 2003; Joormann \& Tran, 2009). Moreover, engaging in rumination following naturally occurring negative life events, predicted better recall of the events at a 2-week follow-up (Conolly \& Alloy, 2018). The interplay of deficient inhibition (which can result in elaborate processing of negative material) and impaired memory control (making this material more difficult to forget), likely creates a cascade of negativity that maintains ruminative thinking over time.

Indeed, theory suggests that inhibition and memory biases do not operate in isolation, but instead interact and influence one another (Hertel \& El-Messidi, 2006; Hirsch, Clark, \& Mathews, 2006). This perspective is consistent with the view of rumination as a habit that encourages people to perceive, interpret, and remember events in a repetitive self-focused manner that enhances negative mood-congruent thinking (Hertel, 2004). In line with these assertions, our previous work has shown that inhibitory biases affect interpretive biases associated with rumination (Daches, Mor, \& Hertel, 2015). Evidence for biased remembering should also be influenced by variation in inhibition; Inhibitory processes should also affect memory biases: deficient inhibition of negative material should make it ultimately more memorable 
than it would be otherwise. This is the prediction that motivated the current experiment.

Several studies found that among dysphoric and depressed individuals, attention biases for negative stimuli were associated with differences in subsequent recall and recognition (Blaut, Paulewicz, Szastok, Prochwicz, \& Koster, 2013; Ellis, Beevers, \& Wells, 2011; Ellis, Wells, Vanderlind, \& Beevers, 2014; Koster, De Raedt, Leyman, \& De Lissnyder, 2010; Wells, Beevers, Robison, \& Ellis, 2010). More relevant to our inquiry, among ruminators, intentional suppression of learned associations poorly facilitated their forgetting (Dieler, Herrmann, \& Fallgatter, 2014; Hertel \& Gerstle, 2003; Hertel, Maydon, Ogilvie, \& Mor, 2018; Joormann \& Tran, 2009). Thus, there is evidence that active suppression of previously attended negative content, a process which relies on inhibitory control, is associated with memory biases among ruminators. In the current study we intended to further investigate this relationship, by examining how the specific nature of attentional bias (i.e., to ignore negative material) has consequences for biases in memory. Therefore, a main goal of the current research is to investigate the causal effect of inhibition on memory by manipulating inhibition of negative information in one task and assessing its effect on memory of negative information in a second task.

CBM research should follow the transfer appropriate framework in designing the degree of overlap between training and transfer tasks (Hertel \& Mathews, 2011). This framework suggests that memory performance is affected by the similarity between the processes occurring during initial exposure and the test. Transfer effects should be expected when the memory task is designed to reflect possible biases in the attention/inhibition task. In our training phase, participants were presented with twoword stimuli (a negative and a neutral one) and learned to ignore one of them. 
Therefore, the question for the subsequent memory task is whether this trained attentional bias would transfer to the encoding stage of the subsequent memory task, in which pairs of pictures were displayed, and participants were asked to select the picture to which they related more. Subsequently, we assessed the relative recall of those pictures. We predicted transfer of the trained inhibition to attention during exposure to the new stimuli in the memory task and, consequently, differential subsequent recall. Thus, we predicted lower negativity bias in recall among participants who were trained to inhibit negative information compared to participants who were trained to attend to such content.

Because ruminators may not be affected by a single session of inhibition training, and the effects of a single session on interpretation bias are small (Daches et al., 2015), in the current experiment we assigned ruminators to five training sessions. Our main goal was to assess training effects on memory biases at post-training. Extending recent findings on sustained effects of rumination-related bias training (Hirsch et al., 2018; Swainston \& Derakshan, 2018; Vermeulen, Brown, Raes, \& Krans, 2018), we examined training effects on inhibition bias, rumination and depressive symptoms in a two-week follow-up. We assessed training effects on rumination using several measures: (a) a trait measure that targets habitual tendencies (Ruminative Response Scale [RRS]; Nolen-Hoeksema \& Morrow, 1991), administered at the two-week follow-up; (b) a state measure that captures momentary fluctuations in ruminative thinking (Momentary Ruminative Self-Focus Inventory; Koster, Marchetti, \& Mor, 2013), administered during each of the training sessions and (c) a measure designed to assess rumination in response to recalling a negative personal event (reactive rumination; Cohen, Mor, \& Henik, 2015; Cohen \& Mor, 2018; Hertel, Mor, Ferrari, Hunt, \& Agrawal, 2014), administered post-training. 
We hypothesized that relative to ruminators who are trained to attend to negative content, those who are trained to inhibit such content would show: (a) better inhibition of negative stimuli on the NAP task (inhibition was assessed at follow-up to avoid dilution of training effects on memory and rumination), (b) reduced negative bias in the memory task, and (c) lower levels of ruminative thinking and depressed mood.

\section{Method}

\section{Participants}

Students at the Hebrew University of Jerusalem (native Hebrew speakers) completed a screening measure of rumination (RRS; Nolen-Hoeksema \& Morrow, 1991). Because we were interested in recruiting only ruminators for this study, only those $(N=85)$ scoring above 10 , which is the median score on the brooding subscale of the RRS, were recruited for the study. All participants had normal or corrected-tonormal vision. Six participants elected not to undergo the training because of time constraints. The remaining 79 participants ( 25 males) were randomly assigned to one of two conditions: (a) Inhibit-negative training (IN) $(n=42)$ and (b) Attend-negative training $(\mathrm{AN})(n=37)$. Participants' mean age was $23.62(S D=2.59)$. Trained, B.A. level, research assistants obtained written informed consents from participants and delivered the entire experimental procedure. Participants received research credit and/or monetary compensation for their participation. The sample size was determined based on an a priori power analysis. For an effect size of .25 - which was based on our previous findings with a similar design (Daches \& Mor, 2014) - we aimed to recruit 62 participants (power .80 ; alpha $=0.05$ ).

\section{General Procedure}

Participants were invited to the lab for a pre-training assessment session and were informed that some of them would be offered the opportunity to participate in a study 
that includes six more lab sessions. In the pre-training assessment session, which lasted about 20 min, participants completed self-report questionnaires and the NAP task (to assess inhibition of negative information). Participants who continued to the training phase of the study were randomly assigned to one of the two conditions (IN or AN). They then completed five training sessions during the 3-week period following the pre-training assessment session. Each training session, which lasted about 15 min, began and ended with a mood and state rumination measure. Immediately following the last training session, participants completed the posttraining assessment that included a memory task and the reactive rumination task (assessing rumination following the recall of a negative personal event). The posttraining session lasted about an hour. Participants were invited to the lab two weeks following training for a follow-up assessment session that lasted about $20 \mathrm{~min}$, in which they completed the NAP task as well as the self-report questionnaires (assessing trait rumination and depression).

\section{The Negative Affective Priming (NAP) Task}

The NAP task is a modification of the Negative Priming task (Neill, 1977) that includes emotional stimuli (Frings, Wentura, \& Holtz, 2007; Joormann, 2006). As illustrated in Figure 1, each trial began with a white fixation cross on a black background presented for $1000 \mathrm{~ms}$ and followed by the stimulus pair. Each stimulus pair was comprised of a prime and a probe display (although participants were not aware of this classification because all displays had the same form). On each display, two words were presented concurrently one above the other, each in a different color (red or blue). The color of the word identified it as a target or distractor. The pair remained on the screen until the participant responded. Participants were asked to evaluate the valence of the target (e.g., the red word) and ignore the distractor. The 
response and its latency were recorded. The location of the target word varied randomly, and the color of the target word (red or blue) was counterbalanced across participants.

Trial types. The NAP task included three blocks of 32 trials. Words appeared once in each block and were repeated across blocks. Each block was comprised of two trial types: inhibition and inhibition control (see Figure 1). On inhibition trials, participants responded to a negatively valenced target following a trial with a negative distractor (i.e. the prime display contained a negative distractor and a neutral target, and the probe display contained a negative target and a neutral distractor). On inhibition control trials, participants also responded to negative targets on the probe displays but the preceding prime displays contained neutral words as both the distractor and the target. To assure that participants do not learn that following a negative distractor they will be asked to respond to a negative target, we distributed filler trials throughout the task. These trials included prime displays that were identical to those in the inhibition and inhibition control trials but probe displays that contained only neutral words. Each trial type appeared 8 times in each block in a random order $(8$ inhibition trials, 8 filler inhibition trials, 8 control inhibition trials and 8 filler control inhibition trials).

Inhibition bias scores were derived by subtracting RTs on probe displays on inhibition control trials (for which the prime display consisted of two neutral words) from RTs on probe displays on inhibition trials (for which the prime display consisted of a neutral target and a negative distractor). This score is an index of the ability to respond to negative stimuli that were previously inhibited as compared to the response to negative stimuli that were not inhibited previously. Positive values indicate better inhibition marked by an increased difficulty to respond to previously inhibited stimuli. 
Stimuli. A set of 32 negative and 96 neutral words were selected for the task; each word was repeated three times across the trials. For selection purposes, 15 independent raters provided valence ratings $(1=$ very negative, $7=$ very positive $)$ for negative and neutral words that ranged from 4 to 6 letters. Words with extreme frequencies of usage in Hebrew were not piloted $(<4$ to a million or $>400$ to a million, Frost \& Plaut, 2005). Negative words were included if all judges rated them as lower than $3(M=1.89, S D=0.36)$ and neutral words were included if all judges rated them between 3 and $5(M=4.07, S D=0.12)$.

\section{Training Procedure}

The training task was similar to the NAP task, whereby each display contained two words and participants were instructed to indicate the valence of a target word (presented in one color) while ignoring the distractor word (presented in a different color). In contrast to the prime-probe structure of the NAP task, in which negative words were targets on half of the trials and distractors on the other half, in the training task, the proportion of negative and neutral targets varied by experimental condition. Thus, in the IN condition, in which participants were trained to ignore negative stimuli and attend to neutral stimuli, negative words were distractors on $85 \%$ of the trials and targets on $15 \%$ of the trials. In the AN condition, in which participants were trained to regard negative words as relevant and ignore neutral stimuli, the proportion of negative and neutral targets was reversed. We used $85 \%$ of the trials rather than $100 \%$ to keep the intent of the training from being transparent and to ensure that participants process the words before pressing on the relevant key.

The randomization schemes of each training session mimicked those used in the NAP task. Forty negative and 46 neutral new words (valence rating; negative: $M=$ 1.65, $S D=0.3 .7$; neutral: $M=4.09, S D=0.18$ ) were selected in the same manner as 
in the NAP task. Word stimuli were identical in the two conditions and were repeated in each session. Each training session lasted approximately 15 min.

In the first training session, participants were given explicit instructions stating that the target word would usually be negative or neutral (depending on condition). In the subsequent four training sessions, standard instructions were given, and participants did not receive any information about the proportion of negative or neutral word targets.

\section{Memory Task}

The memory task consisted of orienting and free-recall phases, separated by a 3min distraction task (backward digit span). In the orienting phase, we displayed 16 pairs of pictures, randomly ordered, and asked participants to select (by pressing a key) the picture in each pair to which they related more. Each picture pair was presented for $6 \mathrm{~s}$, regardless of the timing of the key press, and consisted of a negative, and a neutral picture. The pictures, depicting human figures, were selected from the International Affective Picture System (IAPS; Lang, Bradley, \& Cuthbert, 2008). The negative pictures depicted sad images such as crying people, sickness or loss. The neutral pictures had valence ratings between 4 and 6 and the negative pictures were rated as lower than 4, on Lang and colleagues' (2008), 1 (negative) to 9 (positive) valence rating system of the IAPS pictures. Because the relevant stimulus dimension in this study was valence, we made sure that stimulus arousal levels were low and equal across the training groups. Therefore, arousal level for all selected pictures was 5 or lower, on a scale from 1 (low arousal) to 9 (high arousal). We pilottested picture stimuli to select 16 negative and 16 neutral pictures that were easy to describe in a few words and that their description was distinct from the descriptions of the other pictures. 
In the free recall phase, participants were asked to recall as many of the 32 pictures as possible. Participants wrote verbal descriptions of the pictures, which were matched to the respective pictures by trained research assistants. Inter-rater reliability for matching the descriptions to the respective pictures was excellent $(\mathrm{ICC}=.99)$. Disagreements between raters were resolved by a third rater. Because recall tendencies reflect previous attention and possible elaboration of the selected pictures, we conditionalized recall on selection by calculating the proportion of negative (or neutral) pictures recalled out of the number of negative (or neutral) pictures selected during the orienting task.

\section{Assessment of Ruminative Thinking}

Within-session state rumination. Within-session state-rumination was assessed using the Momentary Ruminative Self-Focus Inventory (Koster et al., 2013), a 6-item visual analog scale (VAS) that ranges from highly agree to highly disagree. Items on this scale measure rumination occurring at the time of assessment (for example: "Right now, I am thinking about the possible meaning of the way I feel"). The inventory has adequate psychometric properties as well as good construct and concurrent validity (Koster et al., 2013) and in the present sample its mean internal consistency was good $(\alpha=.80 ;$ range $=.66-.90)$.

Reactive rumination task. In this procedure, state rumination is assessed following recall of a negative self-relevant memory. As in prior research (Cohen et al., 2014; Hertel et al., 2014) participants were given 4 min. to recall a recent upsetting personal event and then were sked to sit quietly for $2 \mathrm{~min}$, ostensibly having a break from the experiment but really to allow for ruminative thoughts to emerge. Then, they completed a state rumination measure that assessed the degree to which they currently ruminated about the recalled event. This measure includes 10 
rumination-related items modified from the RRS to relate to current thoughts about the event (e.g., "at this moment, I can't stop thinking about what happened"). Participants indicated their response on a VAS that ranged from highly agree to highly disagree. In the present sample, the internal consistency of the reactive rumination measure was good $(\alpha=.79)$.

Trait rumination. Trait rumination was assessed using the RRS (NolenHoeksema \& Morrow, 1991), a 22-item questionnaire that assesses participants' responses to depressed mood. In the present sample, the internal consistency of the RRS was very good $(\alpha=.89)$.

\section{Assessment of Depressive Symptoms and Negative Mood}

Participants completed the Beck Depression Inventory-II (BDI-II; Beck, Steer, \& Brown, 1996). In the present sample, the internal consistency of the BDI-II was very good $(\alpha=.90)$. In addition, on each of the training sessions, participants completed a 6-item mood measure based on the PANAS-X (Watson \& Clark, 1994). This mood measure was intermixed with the within-session state rumination measure, to disguise the purpose of each instrument. The three items measuring negative mood included: depressed, sad and unhappy (mean $\alpha=.93$; range $=.91-.94)$. The three additional items measured positive affect (mean $\alpha=.89$; range $=.88-.91)$.

\section{Results}

\section{Participant Characteristics}

Of the 79 participants who started the training, 75 participants completed all training sessions and the follow-up assessment session, resulting in a drop-out rate of 5\%. Data from 7 participants were removed due to high error rate during training (above 20\%; 3 from the IN condition), and two participants were removed due to outlying scores on the RRS scale (above 2.5 SDs from the mean). Thus, the final 
sample included 66 participants (23 men). Participants in the two conditions did not differ in demographic characteristics and in pre-training levels of rumination and depression (see Table 1).

\section{Training Effects on Inhibition Bias}

Data reduction. Only reaction times (RTs) for probe displays were analyzed. All trials with incorrect responses were excluded $(3.52 \%$ of trials at the pre-training assessment and $6.96 \%$ of trials at the follow-up assessment). Trials involving extreme RTs (longer than $3000 \mathrm{~ms}$ or shorter than $250 \mathrm{~ms} ; 0.95 \%$ of trials at pre-training and $0.54 \%$ at follow-up) were also removed. Finally, RTs $2.5 S D$ above and below the mean RT for each participant were eliminated (1.17\% of trials at pre-training and $1.06 \%$ at follow-up). Thus, in total $5.64 \%$ of responses were eliminated from analyses in the pre-training assessment and $8.57 \%$ of the responses were eliminated in the follow-up assessment.

Change in inhibition from pre-training to follow-up. Inhibition bias scores on the NAP were submitted to a 2 (condition: IN, AN) by 2 (time: pre-training, follow-up) mixed design ANOVA. The interaction of condition by time was marginally significant, $F(1,64)=3.80, p=.06, \eta_{\mathrm{p}}^{2}=.06$. To explore this interaction, we examined simple effects, using Bonferroni-correction, of difference between training conditions within each assessment session. At pre-training, as expected, the two conditions did not differ in inhibition bias $\left(M_{I N}=6.41, S D_{I N}=59.95, M_{A N}=\right.$ 11.29, $\left.S D_{A N}=61.84\right), F(1,64)=.11, p=.75, \eta_{\mathrm{p}}{ }^{2}=.01$. In contrast, at follow-up the difference between conditions was significant, suggesting that inhibition in the IN condition was better than in the AN condition $\left(M_{I N}=24.72, S D_{I N}=50.66, M_{A N}=-\right.$ 11.73, $\left.\left.S D_{A N}=89.65\right), F(1,64)=4.14, p=.05, \eta_{\mathrm{p}}^{2}=.06\right)$.

\section{Training Effects on Memory Bias}


At the post-training assessment session, participants were asked to recall pictures from the preceding orienting phase of 16 displays of neutral-negative picture-pairs in which they were asked to select a picture to which they relate more. Participants in the two experimental conditions did not differ in the number of negative pictures they selected, $t(64)=0.46, p=.65, M_{A N}=8.18, S D_{A N}=1.74, M_{I N}=8.39, S D_{I N}=1.98$, nor in the raw numbers of negative and neutral pictures they recalled (Negative: $t(64)=$ $0.35, p=.73, M_{A N}=8.73, S D_{A N}=2.60, M_{I N}=8.52, S D_{I N}=2.27$, Neutral: $t(64)=0.73$, $\left.p=.47, M_{A N}=6.61, S D_{A N}=2.16, M_{I N}=6.18, S D_{I N}=2.52\right)$.

Our main prediction was that compared to participants in the AN condition who would show preferential recall of negative versus neutral attended images, participants in the IN condition would exhibit reduced negativity bias by showing a reduced preference for recalling negative versus neutral attended images. We submitted the proportion of pictures recalled out of the previously selected to a 2 (condition: IN, AN) by 2 (picture type: negative, neutral) mixed-design ANOVA. Means are shown in Figure 2. Across conditions, participants recalled proportionally more negative than neutral pictures they selected during the orientation phase, $F(1$, $63)=17.99, p<.001, \eta_{\mathrm{p}}{ }^{2}=.22$. As predicted, this main effect was qualified by a condition by type interaction, $F(1,63)=4.52, p=.04, \eta_{\mathrm{p}}^{2}=.07$. To explore this interaction, we examined simple effects, using Bonferroni-correction, of valence differences within each condition. In the AN condition, participants recalled more negative compared to neutral pictures, $F(1,63)=19.96 p<.001, \eta_{\mathrm{p}}{ }^{2}=.24$, whereas in the IN condition participants recalled negative and neutral pictures equally, $F(1,63)=$ $2.28, p=.14, \eta_{\mathrm{p}}^{2}=.24$

\section{Training Effects on Rumination}


Within-session state rumination. Within-session state rumination scores assessed at the beginning of each training session were submitted to a 2 (condition: IN, AN) by 5 (session) mixed-design ANOVA. The interaction between session and condition was significant, $\left.F(4,56)=2.82, p=.03, \eta_{\mathrm{p}}^{2}=.17\right)$. To explore this interaction, we examined simple effects, using Bonferroni-correction, of differences among assessment points within each condition. As predicted (see Table 2), in the IN condition there was a significant reduction in state rumination across assessment points, $F(4,56)=4.42, p<.01, \eta_{\mathrm{p}}^{2}=.24$. In the $\mathrm{AN}$ condition too, state rumination changed throughout training but to a lesser degree, $F(4,56)=2.54, p=.05, \eta_{\mathrm{p}}{ }^{2}=.15$.

Reactive rumination. State rumination scores, potentially influenced by the recall of a negative life event at the post-training assessment session, were compared across conditions by using an independent samples $t$-test. The difference between experimental conditions was significant $\left(M_{I N}=51.05, S D_{I N}=15.57, M_{A N}=60.71\right.$, $S D_{A N}=20.44, t(63)=2.11, p=.04$, Cohen's $\left.d=.53\right)$. As expected, reactive rumination was higher in the AN than in the IN condition.

Trait rumination. RRS scores were submitted to a 2 (condition: IN, AN) by 2 (time: pre-training, follow-up) mixed-design ANOVA. Both the main effect for time and the interaction between time and condition were non-significant $(p>.10)$. Thus, training-congruent effects on rumination were observed in reactive rumination and within-session ruminative thinking, but not in trait rumination.

\section{Training Effects on Negative Mood and Depressive Symptoms}

We assessed the effect of training on negative mood in two ways: (a) change in depressive symptoms from pre-training to follow-up, as revealed by BDI scores, (b) change in negative mood throughout the training sessions using the mood rating VAS administered at each training session. The condition-by-time interaction effect on BDI 
(BDI: $\left.F(1,64)=0.43, p=.84, \eta_{\mathrm{p}}^{2}<.001\right)$ and the condition-by-session interaction effect on negative mood, $\left(F(4,56)=0.60, p=.66, \eta_{\mathrm{p}}{ }^{2}=.04\right)$ were both nonsignificant.

\section{Discussion}

This study replicates and extends our prior findings concerning the effect of training inhibition of negative content. One way in which we extend prior findings is by assessing whether training effects on inhibition are sustained following termination of training delivery. Although several studies have examined the effects of CBM at follow-up (Blackwell \& Holmes, 2010; Lang, Moulds \& Holmes, 2009; Schmidt, Richey, Buckner \& Timpano, 2009; Wells \& Beevers, 2010), the effects of inhibition training on rumination have only been assessed immediately following the training. The current findings cautiously suggest that training effects on inhibition are maintained two weeks following the training. These effects were obtained using a different set of emotional stimuli than those used for training, suggesting generalization of training. Our findings, although small in magnitude, support the effectiveness of CBM in resisting rumination by modifying the inhibition of negative material, a specific cognitive bias assumed to be causally involved in rumination.

Our main prediction concerned the transfer of the training effects to biased recall. Participants who were trained to inhibit negative content, later showed a reduced negativity bias, manifested in lower preference for recalling negative versus neutral images to which they attended. Thus, trained inhibition transferred to the encoding stage of the memory task, consequently affecting the subsequent recall. Clearly, because participants in the training condition were encouraged to inhibit negative information at the same time as they were encouraged to process neutral information, the two cannot be teased apart. Similar findings were found when depressed 
participants were instructed to use new targets to help keep them from thinking about the previously learned negative targets. Specifically, depressed participants were able to use neutral substitutes to aid them in forgetting negative content (Joormann, Hertel, LaMoult, \& Gotlib, 2009). Future research should delineate features of helpful alternative percepts or alternative processing options. For example, a more effective inhibition training may include positive targets as substitutes.

Our findings are in line with prior research that showed that inhibitory mechanisms may form the basis of negative biases in memory (Storm \& Jobe, 2012). They also add to our previous findings that inhibition plays a role in interpretation biases (Daches et al., 2015). Thus, consistent with the combined cognitive bias hypothesis (Hirsch et al., 2006), the habitual use of rumination may be associated not only with how one attends to or interprets emotion-eliciting events but also with how such events are remembered later. This combination of biases may increase the likelihood of repetitive negative thoughts, trapping ruminators in a vicious cycle that maintains and exacerbates negative affect and can enhance depressive symptoms (for a review see de Raedt, Hertel, \& Watkins, 2015). Moreover, difficulty inhibiting negative content as well as biased interpretation and recall of mood-incongruent information may interfere with the use of more adaptive emotion regulation strategies such as cognitive reappraisal (Cohen $\&$ Mor, 2018). However, caution should be taken when interpreting our training effects on memory as indicative of inhibition because inhibition processes in the memory task can only be inferred, but were not measured directly. Alternative explanations for the group differences in memory should be explored in future research.

Training inhibition had consequences for ruminative thinking within session and following the recall of a negative personal event (reactive rumination), but not for trait 
rumination. The differential effects may be attributed to inherent differences between measures of trait and state rumination (e.g., Watkins \& Nolen-Hoeksema, 2014). Whereas state rumination measures examine concurrent ruminative responses to a temporary and specific situation, trait rumination measures denote a stable tendency to respond with ruminative thinking across situations. The null effects of training on a measure of trait rumination may point to the difficulty to eliminate established ruminative habits. It is possible that in order to affect a stable trait, $\mathrm{CBM}$ should be more extensive and perhaps tackle a number of cognitive biases. Another possibility is that the lack of effect on trait rumination reflects the constraints of the RRS, the measure we used to assess trait rumination (e.g., Griffith \& Raes, 2014). The RRS requires participants to indicate what they generally do when they feel sad, and participants' retrospective and non-specific report may be vulnerable to bias and distortion (Stone et al., 1998). Responding to the RRS also requires participants to notice habitual changes that may not be noticeable in a short time frame of examination. Because ruminative thoughts occur in varied circumstances, it may be useful to assess the effects of CBM on rumination in real life, using diary methodology (e.g., Hoorelbeke, Koster, Demeyer, Loeys, \& Vanderhasselt, 2016; Moberly \& Watkins, 2008). Such an approach would be consistent with our findings on the within-session and reactive rumination measures.

Several limitations should be taken into account. First, the relatively small sample size and low statistical power precluded us from conducting mediation analyses to explore whether training inhibition of negative information affects ruminative thinking via its effect on memory. Second, our sample was not characterized by significant levels of depressive symptoms. This fact prevented the examination of possible therapeutic effects of our procedure. This limitation might 
also explain why, contrary to our predictions, the training did not affect depressive symptoms and negative mood. Third, we assessed inhibition at the follow-up assessment point but not immediately post-training. Because in the assessment task, negative words are targets on half of the trials, presentation of this task at the end of the training would have diluted training effects on the follow-up assessment. This design choice made it impossible to examine whether training leads to group differences in inhibition of negative stimuli which in turn contribute to group differences in rumination and memory biases at post-training. Fourth, it is possible that the verbal stimuli used in the training paradigm do not elicit a strong enough emotional effect. This suggestion is supported by the fact that our previous effort to detect mood effects were similarly unsuccessful (Daches et al., 2015), but using pictorial stimuli in a similar training task did yield effects on mood (Cohen et al., 2015). Finally, the two-week follow-up assessed in this study is relatively short and future studies should examine whether training effects are sustained across longer time frames.

Despite these limitations, our results help shed light on a central cognitive mechanism of ruminative habits, the inhibition of negative information and its association with memory biases that characterize rumination. Moreover, our findings highlight the importance of interventions that focus on modifying cognitive biases as a possible way to help people stop ruminative habits, ultimately, with the goal of preventing psychopathology and particularly depression. Future studies should consider designing more ecologically valid interventions incorporating training paradigms in dynamic and complex environments. 


\section{References}

Aldao, A., Nolen-Hoeksema, S., \& Schweizer, S. (2010). Emotion-regulation strategies across psychopathology: A meta-analytic review. Clinical psychology review, 30, 217-237.

Anderson, M. C., \& Green, C. (2001). Suppressing unwanted memories by executive control. Nature, 410, 366.

Beck, A. T., Steer, R. A., \& Brown, G. K. (1996). Manual for the Beck Depression Inventory-II, San Antonio, TX: Psychological Corporation.

Beevers, C. G., Clasen, P. C., Enock, P. M., \& Schnyer, D. M. (2015). Attention bias modification for major depressive disorder: Effects on attention bias, resting state connectivity, and symptom change. Journal of abnormal psychology, 124(3), 463.

Blackwell, S. E., \& Holmes, E. A. (2010). Modifying interpretation and imagination in clinical depression: A single case series using cognitive bias modification. Applied Cognitive Psychology, 24, 338-350.

Blaut, A., Paulewicz, B., Szastok, M., Prochwicz, K., \& Koster, E. (2013). Are attentional bias and memory bias for negative words causally related?. Journal of Behavior Therapy and Experimental Psychiatry, 44, 293-299.

Cousineau D. (2005) Confidence intervals in within-subject designs: A simpler solution to Loftus and Masson's method. Tutorial in Quantitative Methods for Psychology, 1, 42-45.

Cohen, N., \& Mor, N. (2018). Enhancing Reappraisal by Linking Cognitive Control and Emotion. Clinical Psychological Science, 6, 155-163. 
Cohen, N., Mor, N., \& Henik, A (2014). Linking executive control and emotional response - A training procedure to reduce rumination. Clinical Psychological Science, 1-11.

Connolly, S. L., \& Alloy, L. B. (2018). Negative event recall as a vulnerability for depression: Relationship between momentary stress-reactive rumination and memory for daily life stress. Clinical Psychological Science, 6, 32-47.

Daches, S., \& Mor, N. (2014). Training ruminators to inhibit negative information: A preliminary report. Cognitive Therapy and Research, 38, 160-171.

Daches, S., Mor, N., \& Hertel, P. (2015). Rumination: Cognitive consequences of training to inhibit the negative. Journal of Behavior Therapy and Experimental Psychiatry, 49A, 76-83.

De Raedt, R., Hertel, P. T., \& Watkins, E. R. (2015). Mechanisms of repetitive thinking: introduction to the special series. Clinical Psychological Science, 3(4), 568-573.

Dieler, A. C., Herrmann, M. J., \& Fallgatter, A. J. (2014). Voluntary suppression of thoughts is influenced by anxious and ruminative tendencies in healthy volunteers. Memory, 22, 184-193.

Demeyer, I., De Lissnyder, E., Koster, E. H., \& De Raedt, R. (2012). Rumination mediates the relationship between impaired cognitive control for emotional information and depressive symptoms: A prospective study in remitted depressed adults. Behaviour Research and Therapy, 50, 292-297.

Ellis, A. J., Beevers, C. G., \& Wells, T. T. (2011). Attention allocation and incidental recognition of emotional information in dysphoria. Cognitive Therapy and Research, 35(5), 425-433. 
Ellis, A. J., Wells, T. T., Vanderlind, W. M., \& Beevers, C. G. (2014). The role of controlled attention on recall in major depression. Cognition and Emotion, 28(3), 520-529.

Engen, H. G., \& Anderson, M. C. (2018). Memory control: A fundamental mechanism of emotion regulation. Trends in Cognitive Sciences, 22, 982-995.

Fawcett, J. M., Benoit, R. G., Gagnepain, P., Salman, A., Bartholdy, S., Bradley, C., ... \& Anderson, M. C. (2015). The origins of repetitive thought in rumination: Separating cognitive style from deficits in inhibitory control over memory. Journal of behavior therapy and experimental psychiatry, 47, 1-8.

Friedman, N. P., \& Miyake, A. (2004). The relations among inhibition and interference control functions: A latent-variable analysis. Journal of Experimental Psychology: General, 133, 101-135.

Frings, C., Wentura, D., \& Holtz, M. (2007). Dysphorics cannot ignore unpleasant information. Cognition and Emotion, 21, 1525-1534.

Frost, R., \& Plaut, D. (2005). The word-frequency database for printed Hebrew. Retrieved from http:// word-freq.mscc.huji.ac.il/index.html

Griffith, J. W., \& Raes, F. (2014). Factor Structure of the Ruminative Responses Scale. European Journal of Psychological Assessment, 31, 247.

Hasher, L., \& Zacks, R. T. (1988). Working memory, comprehension, and aging: A review and a new view. Psychology of Learning and Motivation, 22, 193-225.

Hertel, P. T. (1997). On the contribution of deficient cognitive control to memory impairment in depression. Cognition and Emotion, 11, 569-583.

Hertel, P. T. (2004). Memory for emotional and non-emotional events in depression: A question of habit. In D. Reisberg \& P. Hertel (Eds.), Memory and emotion (pp. 186-216). New York, NY: Oxford University Press. 
Hertel, P. T., \& El-Messidi, L. (2006). Am I blue? Depressed mood and the consequences of self-focus for the interpretation and recall of ambiguous words. Behavior therapy, 37, 259-268.

Hertel, P. T., \& Gerstle, M. (2003). Depressive deficits in forgetting. Psychological Science, 14, 573-578.

Hertel, P. T., \& Mathews, A. (2011). Cognitive bias modification: Past perspectives, current findings, and future applications. Perspectives on Psychological Science, 6, 521-536.

Hertel, P. T., Maydon, A., Cottle, J., \& Vrijsen, J. N. (2016). Cognitive Bias Modification Retrieval Practice to Simulate and Oppose Ruminative Memory Biases. Clinical Psychological Science, 5, 122-130.

Hertel, P. T., Maydon, A., Ogilvie, A., \& Mor, N. (2018). Ruminators (unlike others) fail to show suppression-induced forgetting on indirect measures of memory. Clinical Psychological Science, 6, 872-881.

Hertel, P., Mor, N., Ferrari, C., Hunt, O., \& Agrawal, N. (2014). Looking on the Dark Side Rumination and Cognitive-Bias Modification. Clinical Psychological Science, 2, $714-726$.

Hirsch, C. R., Clark, D. M., \& Mathews, A. (2006). Imagery and interpretations in social phobia: Support for the combined cognitive biases hypothesis. Behavior Therapy, 37, 223-236.

Hirsch, C. R., Krahé, C., Whyte, J., Loizou, S., Bridge, L., Norton, S., \& Mathews, A. (2018). Interpretation training to target repetitive negative thinking in Generalized Anxiety Disorder and Depression. Journal of Consulting and Clinical Psychology, 86, 1017-1030. 
Hoorelbeke, K., Koster, E. H., Demeyer, I., Loeys, T., \& Vanderhasselt, M. A. (2016). Effects of cognitive control training on the dynamics of (mal) adaptive emotion regulation in daily life. Emotion, 16, 945-956.

Joormann, J. (2006). Differential effects of rumination and dysphoria on the inhibition of irrelevant emotional material: Evidence from a negative priming task. Cognitive Therapy and Research, 30, 149-160.

Joormann, J. Hertel, P. T., LeMoult, J., \& Gotlib, I. (2009). Training forgetting of negative material in depression. Journal of Abnormal Psychology, 118, 34-43.

Joormann, J., \& Tran, T. B. (2009). Rumination and intentional forgetting of emotional material. Cognition and Emotion, 23, 1233-1246.

Koster, E. H., De Raedt, R., Leyman, L., \& De Lissnyder, E. (2010). Mood-congruent attention and memory bias in dysphoria: exploring the coherence among information-processing biases. Behaviour Research and Therapy, 48(3), 219225.

Koster, E. H., De Lissnyder, E., Derakshan, N., \& De Raedt, R. (2011). Understanding depressive rumination from a cognitive science perspective: The impaired disengagement hypothesis. Clinical psychology review, 31, 138145.

Koster, E. H. W., Marchetti, I., \& Mor, N. (2013, September). The Momentary Ruminative Self-focus Inventory (MRSI): Validation and psychometric evaluation. Paper presented at the meeting of the European Association of Behavioural and Cognitive Therapies, Marrakech, Morocco.

Koster, E. H., Hoorelbeke, K., Onraedt, T., Owens, M., \& Derakshan, N. (2017). Cognitive control interventions for depression: a systematic review of findings from training studies. Clinical Psychology Review, 53, 79-92. 
Lang, P. J., Bradley, M. M., \& Cuthbert, B. N. (2008). International affective picture system (IAPS): Affective ratings of pictures and instruction manual. Technical Report A-8. University of Florida, Gainesville, FL. Lang, T. J., Moulds, M. L., \& Holmes, E. A. (2009). Reducing depressive intrusions via a computerized cognitive bias modification of appraisals task: Developing a cognitive vaccine. Behaviour Research and Therapy, 47, 139-145.

Linville, P. (1996). Attention inhibition: Does it underlie ruminative thought. Advances in social cognition, 9, 121-133.

Lyubomirsky, S., Caldwell, N. D., \& Nolen-Hoeksema, S. (1998). Effects of ruminative and distracting responses to depressed mood on retrieval of autobiographical memories. Journal of Personality and Social Psychology, 75, 166-177.

Moberly, N. J., \& Watkins, E. R. (2008). Ruminative self-focus, negative life events, and negative affect. Behaviour Research and Therapy, 46, 1034-1039.

Morey R. D. (2008) Confidence intervals from normalized data: A correction to Cousineau (2005). Tutorial in Quantitative Methods for Psychology, 4, 61-64.

Moulds, M. L., Kandris, E., \& Williams, A. D. (2007). The impact of rumination on memory for self-referent material. Memory, 15, 814-821.

Neill, W. T. (1977). Inhibitory and facilitatory processes in selective attention. Journal of Experimental Psychology: Human Perception \& Performance, 3 , $444-450$.

Nolen-Hoeksema, S., \& Morrow, J. (1991). A prospective study of depression and posttraumatic stress symptoms after a natural disaster: The 1989 Loma Prieta earthquake. Journal of Personality and Social Psychology, 611, 115-121. 
Nolen-Hoeksema, S., Wisco, B. E., \& Lyubomirsky, S. (2008). Rethinking rumination. Perspectives on psychological science, 3, 400-424.

Schmidt, N. B., Richey, J. A., Buckner, J. D., \& Timpano, K. R. (2009). Attention training for generalized social anxiety disorder. Journal of Abnormal Psychology, 118, 5.

Stone, A. A., Schwartz, J. E., Neale, J. M., Shiffman, S., Marco, C. A., Hickcox, M., ... \& Cruise, L. J. (1998). A comparison of coping assessed by ecological momentary assessment and retrospective recall. Journal of Personality and Social Psychology, 74, 1670.

Storm, B. C., \& Jobe, T. A. (2012). Retrieval-induced forgetting predicts failure to recall negative autobiographical memories. Psychological Science, 23, 13561363.

Swainston, J., \& Derakshan, N. (2018). Training cognitive control to reduce emotional vulnerability in breast cancer. Psycho-Oncology, 27, 1780-1786.

Vermeulen, M., Brown, A. D., Raes, F., \& Krans, J. (2018). Decreasing Event Centrality in Undergraduates Using Cognitive Bias Modification of Appraisals. Cognitive Therapy and Research, 1-12.

Watson, D., \& Clark, L. A. (1994). The PANAS-X: Manual for the positive and negative affect schedule-expanded form. Ames: The University of Iowa.

Watkins, E. R., \& Nolen-Hoeksema, S. (2014). A habit-goal framework of depressive rumination. Journal of Abnormal Psychology, 123, 24-34.

Wells, T. T., \& Beevers, C. G. (2010). Biased attention and dysphoria: Manipulating selective attention reduces subsequent depressive symptoms. Cognition and Emotion, 24, 719-728. 
Wells, T. T., Beevers, C. G., Robison, A. E., \& Ellis, A. J. (2010). Gaze behavior predicts memory bias for angry facial expressions in stable dysphoria. Emotion, 10, 894.

Whitmer, A. J., \& Gotlib, I. H. (2013). An attentional scope model of rumination. Psychological Bulletin, 139(5), 1036.

Yang, Y., Cao, S., Shields, G. S., Teng, Z., \& Liu, Y. (2017). The relationships between rumination and core executive functions: A meta-analysis. Depression and Anxiety, 34, 37-50.

Zetsche, U., \& Joormann, J. (2011). Components of interference control predict depressive symptoms and rumination cross-sectionally and at six months follow-up. Journal of Behavior Therapy and Experimental Psychiatry, 42, $65-73$. 
Table 1

Demographic Characteristics and Means and Standard Deviations of all Measures at the Pre-Training Assessment

\begin{tabular}{lll}
\hline & IN $(n=33)$ & AN $(n=33)$ \\
\hline Age & $23.18(1.94)$ & $24.21(3.14)$ \\
Gender ratio (F/M) & $20 / 13$ & $23 / 10$ \\
BDI & $12.24(8.48)$ & $10.64(6.44)$ \\
RRS & $47.64(8.43)$ & $44.03(7.58)$ \\
Brooding & $12.97(2.37)$ & $11.97(1.78)$ \\
\end{tabular}

Note. $\mathrm{IN}=$ Inhibit-negative training; $\mathrm{AN}=$ Attend-negative training; $\mathrm{BDI}=$ Beck

Depression Inventory-II; RRS = Ruminative Responses Scale; 
Running head: TRAINING INHIBITION AFFECTS RUMINATION AND MEMORY 30

Table 2

Within-Session State Rumination Scores: Means and Standard Deviations

\begin{tabular}{lll}
\hline & IN $(n=33)$ & AN $(n=33)$ \\
\hline Day1 & $55.05(15.38)$ & $50.69(17.51)$ \\
Day2 & $45.43(15.94)$ & $44.98(18.62)$ \\
Day3 & $48.02(15.71)$ & $42.59(20.60)$ \\
Day4 & $41.21(17.38)$ & $44.79(24.73)$ \\
Day5 & $43.76(18.49)$ & $48.16(23.87)$ \\
\hline
\end{tabular}

Note. IN = Inhibit-negative training; $\mathrm{AN}=$ Attend-negative training 


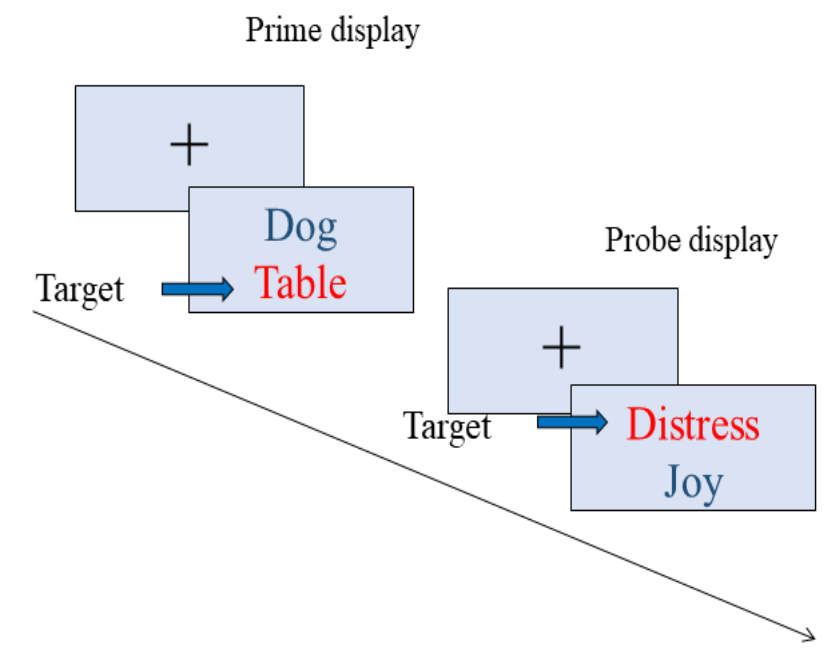

Control inhibition trial

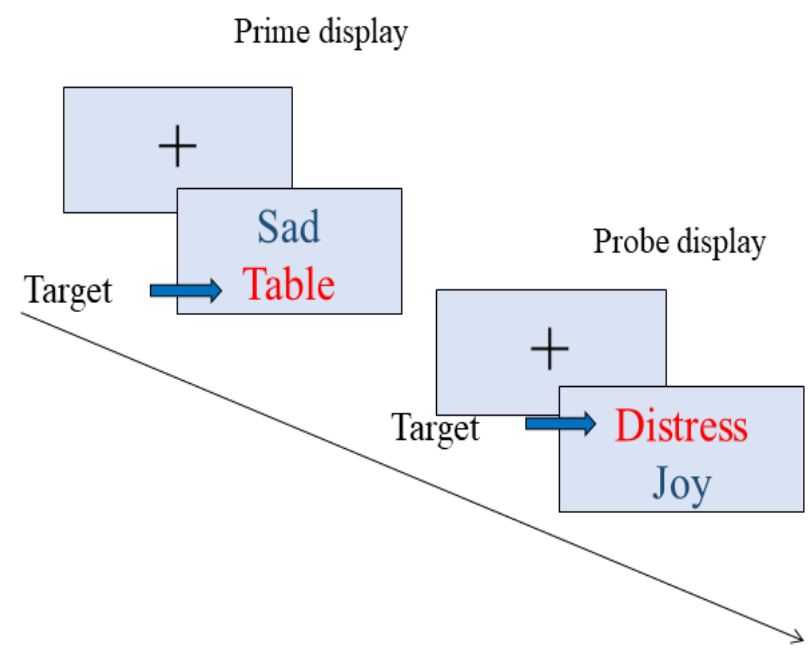

Inhibition trial

Figure 1. The negative affective priming task (NAP): example of trial types 


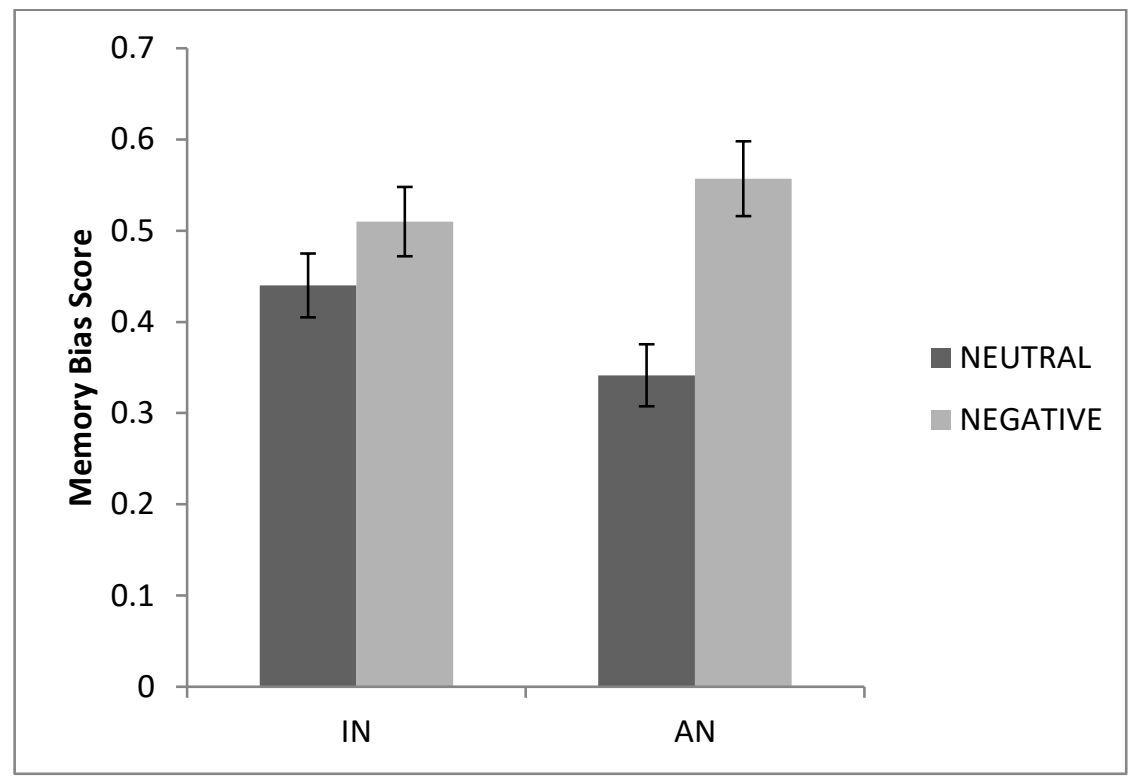

Figure 2. Proportion of neutral and negative pictures recalled out of the neutral and negative pictures participants selected to attend to in the inhibit-negative (IN) and attend-negative (AN) training conditions. Error bars represent confidence intervals (95\%) for within-subject comparisons, calculated according to Cousineau (2005) and Morey (2008). 